\title{
Low Power Upconversion Mixer for Medical Remote Sensing
}

\author{
De Xing Lioe, ${ }^{1}$ Suhaidi Shafie, ${ }^{1,2}$ Harikrishnan Ramiah, ${ }^{3}$ and Gim Heng Tan ${ }^{1,4}$ \\ ${ }^{1}$ Department of Electrical and Electronics Engineering, Faculty of Engineering, Universiti Putra Malaysia, \\ 43400 Serdang, Selangor, Malaysia \\ ${ }^{2}$ Institute of Advanced Technology (ITMA), Universiti Putra Malaysia, 43400 Serdang, Selangor, Malaysia \\ ${ }^{3}$ Department of Electrical Engineering, Faculty of Engineering, University of Malaya, 50603 Kuala Lumpur, Malaysia \\ ${ }^{4}$ Department of Electrical and Electronic Engineering, Segi University, 47810 Petaling Jaya, Selangor, Malaysia \\ Correspondence should be addressed to Harikrishnan Ramiah; hrkhari@um.edu.my
}

Received 5 March 2014; Revised 6 June 2014; Accepted 11 June 2014; Published 8 July 2014

Academic Editor: Mohammad Tawfik

Copyright (C) 2014 De Xing Lioe et al. This is an open access article distributed under the Creative Commons Attribution License, which permits unrestricted use, distribution, and reproduction in any medium, provided the original work is properly cited.

\begin{abstract}
This work presents the design of a low power upconversion mixer adapted in medical remote sensing such as wireless endoscopy application. The proposed upconversion mixer operates in ISM band of $433 \mathrm{MHz}$. With the carrier power of $-5 \mathrm{dBm}$, the proposed mixer has an output inferred $1 \mathrm{~dB}$ compression point of $-0.5 \mathrm{dBm}$ with a corresponding output third-order intercept point (OIP3) of $7.1 \mathrm{dBm}$. The design of the upconversion mixer is realized on CMOS $0.13 \mu \mathrm{m}$ platform, with a current consumption of $594 \mu \mathrm{A}$ at supply voltage headroom of $1.2 \mathrm{~V}$.
\end{abstract}

\section{Introduction}

Mixer is the lifeline of the upconversion process in a transmitter, coupled with the integrating voltage controlled oscillator (VCO) and power amplifiers (PA) circuit defining the transmitter. An upconversion mixer is tasked upon performing frequency translation from a baseband frequency to radio frequency (RF). The supply voltage headroom limitation aligned with the scaling of CMOS technology has initiated efforts upon the realization of low power architecture deemed crucial in portable wireless electronic devices in prolonging the battery lifetime. The limitation of voltage headroom and the degradation of the performance at high operating frequency arise concurrently with technology scaling [1].

The highlight of performance indicator for upconversion mixer design is the linearity and power consumption. Passive mixers do provide comparatively superior linearity performance in comparison to an active architecture, with a penalty paid in conversion loss, often requiring a mandatory larger carrier signal power. Large carrier signal power translates to higher power consumption, while low carrier signal power is undesirable for passive mixer integration $[2,3]$. Gilbert cell mixer is the default conventional double balanced architecture in radio frequency integrated circuit (RFIC) realization. The advantage of Gilbert cell mixer is in its superior port-to-port isolation and its ability in cancelling off undesired RF and local oscillator (LO) output signal feedthrough in the midst of providing higher conversion gain. In the evolution of technology scaling with a proportional lower supply headroom the fact that Gilbert cell mixers integrate high number of series stacked transistors limits the endeavor in achieving low power consumption, [4-11].

Various architectures had been proposed to combat the constraint of voltage headroom limitation [7-9]. Foldedswitching is a promising alternative in operating at low supply voltage abstaining significance effect on the gain and linearity performance [1]. However, with an additional integration of biasing current source, noise is added into the circuit, while the power consumption is ramped up. An alternative solution for low voltage headroom implementation is the switchedtransconductance, where the switching stage and transconductance stage are swapped in position respective to the conventional double-balanced mixer [4]. This architecture solves the setback of stacking transistors, while upholding the performances with lower power consumption. 
The switching pairs of the transconductance stage are turned on and off alternately. The principle of frequency translation is akin to double-balanced topology. The aspect ratio in the width of PMOS to the width of NMOS is twice in magnitude justifying different carrier mobility between PMOS and NMOS devices. At node $\langle a\rangle$ of Figure 2, for a positive half cycle of LO signal, $M_{3}$ is switched off while $M_{1}$ is switched on, resulting in the transconductor to be switched on. The reversed operation is observed at node $\langle b\rangle$. Push pull configuration of switching stage is adopted instead of single NMOS switch due to larger output swing and thus providing superior noise immunity.

This paper presents the design of low power switched transconductance mixer targeted towards portable medical devices at $433 \mathrm{MHz}$ operating frequency, simulated in $0.13 \mu \mathrm{m}$ CMOS technology. The operating frequency of $433 \mathrm{MHz}$ is selected in consideration of the in-body wireless communication losses. The range of frequency with least losses is found to be between $400 \mathrm{MHz}$ and $900 \mathrm{MHz}$ [12], which confirms the industrial, scientific, and medical (ISM) band with frequency ranging from $433.05 \mathrm{MHz}$ to $434.79 \mathrm{MHz}$, and the medical implant communication services (MICS) with frequency of $402-405 \mathrm{MHz}$. ISM band is chosen as the preferred operating frequency over MICS although the latter is reserved by Federal Communications Commission (FCC) for implantable medical devices as MICS has low data rate up to a permissible level of $500 \mathrm{kbps}$.

The paper is organized as follows. Section 2 discusses the architecture and operation of the proposed upconversion mixer. The simulation result is presented in Section 3 in verifying the performance. Finally the conclusion is given in Section 4.

\section{Mixer Architecture and Operation}

Figure 1 shows the architecture of the conventional doublebalanced mixer encapsulating the transconductance stage $\left(M_{1}, M_{2}\right)$, switching stage $\left(M_{3}-M_{6}\right)$, and the output load resistor, $R_{L}$.

The transconductance stage converts the input voltage to current signal, which is subjected to mix with LO or carrier signal in the switching stage. A modified structure of the double-balanced mixer in Figure 1 is proposed in Figure 2 which consists of switching pairs $\left(M_{1}-M_{4}\right)$, transconductance stage $\left(M_{5}-M_{8}\right)$, and load stage $\left(M_{9}, M_{10}\right.$ and $\left.R_{L}\right)$ coupled with the compensating common-mode feedback structure (CMFB). $M_{11}$ is the tail transistor integrating as current source. In comparison with the conventional architecture, the inverting switching stage and transconductance stage are swapped in position.

CMFB structure is adapted where the PMOS transistor $M_{9}$ and $M_{10}$ operates as current source for the differential signals. Common mode current absorbed by the two PMOS load transistor increases the differential gain by

$$
A_{v}=-g_{m}\left(R_{L}\left\|r_{o 1}\right\| r_{o 2}\right)
$$

and at the same time allowing wider voltage headroom. It is an encouraging alternative in integrating diode-connected

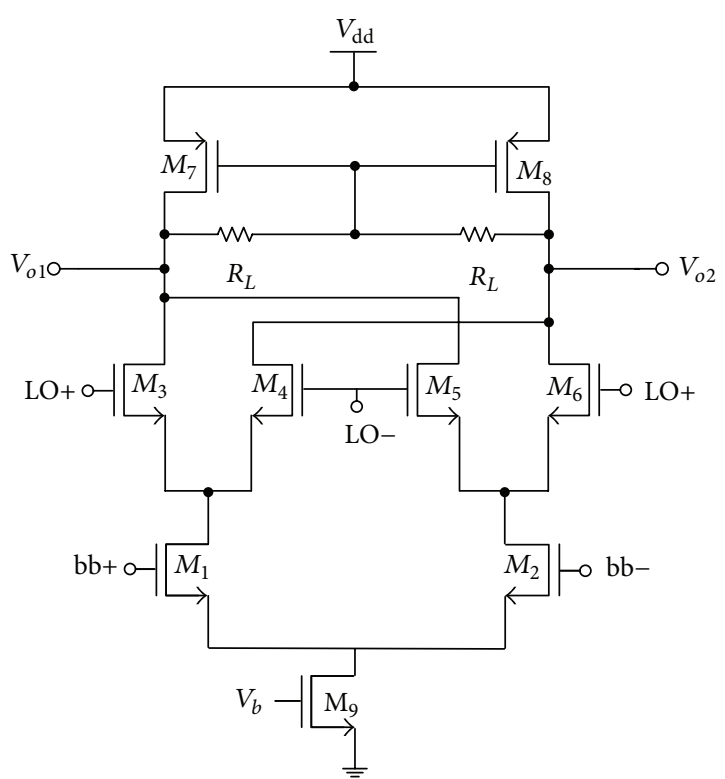

Figure 1: Conventional double-balanced mixer.

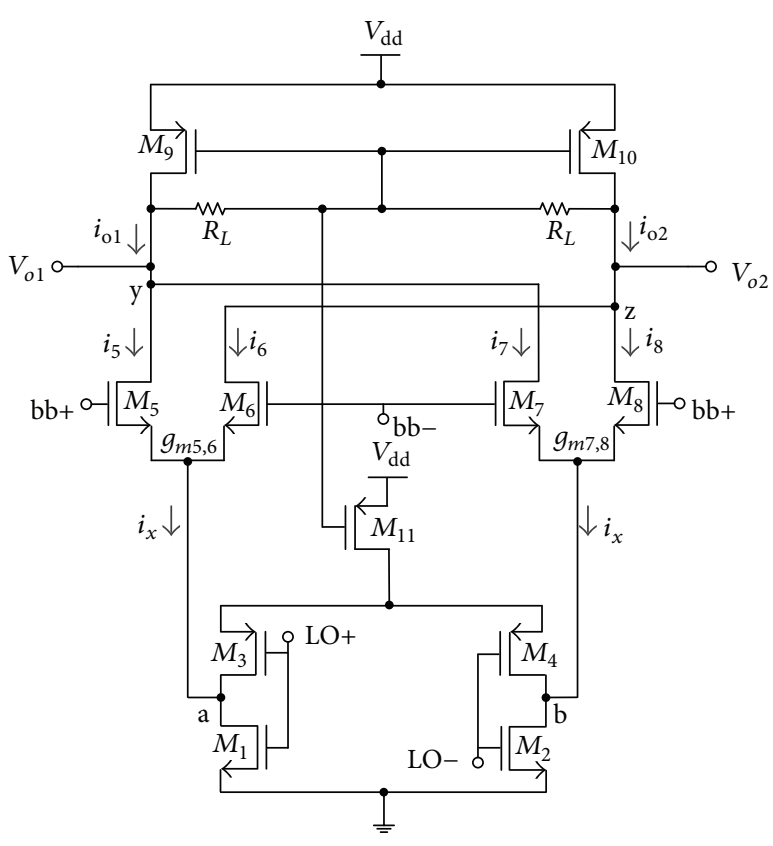

Figure 2: Proposed upconversion mixer.

PMOS load rather than resistor loading, even in the event that the advantages of CMFB comes with a trade-off of higher noise introduced to the circuit. The rational of this tradeoff is constructed, such that the noise figure is not paramount in upconversion mixer [5], and the significance of low power consumption in the application of portable medical devices is inevitable.

Transistor $M_{11}$ provides dc stability for the mixer integrating as a current source for the switching pair. The biasing voltage of $M_{11}$ is fed from CMFB regulating the output voltage and eliminating the need of a redundant bias voltage 


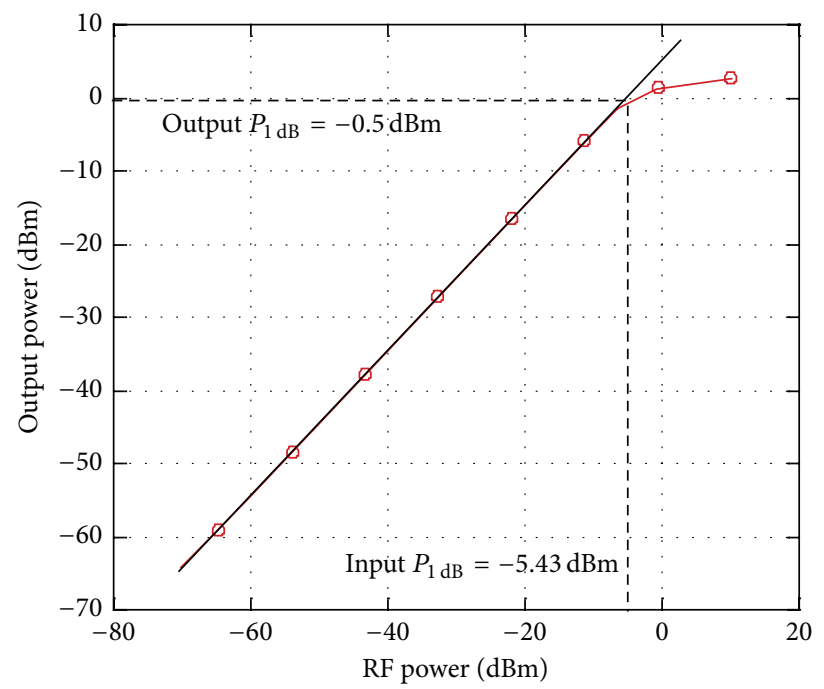

(a)

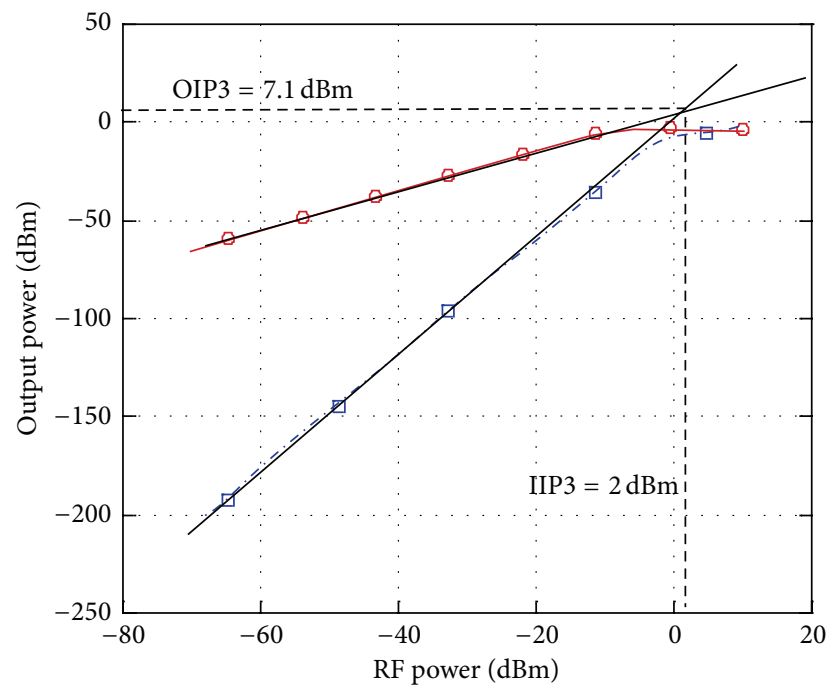

(b)

Figure 3: (a) $1 \mathrm{~dB}$ compression point. (b) Third-order intercept point.

network, thus relaxing the complexity and active chip area consumption.

The comprehensive operation of the proposed mixer as illustrated in Figure 2 is depicted by the following mathematical expressions. Current through transistor $M_{5}$ is

$$
i_{5}=g_{m_{5,6}} \cdot \frac{1}{2} v_{\mathrm{bb}} \cdot \frac{1}{2} i_{x}=\frac{1}{4} g_{m_{5,6}} v_{\mathrm{bb}} i_{x},
$$

where $g_{m_{5,6}}$ is the transconductance for NMOS transistor pair $M_{5}$ and $M_{6}, v_{\mathrm{bb}}=V_{\mathrm{bb}} \sin \omega_{\mathrm{bb}} t$ is the input baseband signal, and $i_{x}$ is the current due to instantaneous LO voltage. Similarly, current through transistor $M_{6}$ can be expressed as

$$
i_{6}=g_{m_{5,6}} \cdot-\frac{1}{2} v_{\mathrm{bb}} \cdot \frac{1}{2} i_{x}=-\frac{1}{4} g_{m_{5,6}} v_{\mathrm{bb}} i_{x} .
$$

Current at node $\langle y\rangle$ can then be obtained as

$$
i_{o 1}=\frac{1}{2} g_{m_{5,6}} v_{\mathrm{bb}} i_{x}
$$

Similarly, current at node $\langle z\rangle$ is expressed as

$$
i_{o 2}=-\frac{1}{2} g_{m_{7,8}} v_{\mathrm{bb}} i_{x}
$$

The differential mixer output current can be derived as

$$
\begin{aligned}
i_{\text {out }} & =i_{o 1}-i_{o 2} \\
& =\frac{1}{2} g_{m_{5,6}} v_{\mathrm{bb}} i_{x}-\left(-\frac{1}{2} g_{m_{7,8}} v_{\mathrm{bb}} i_{x}\right)
\end{aligned}
$$




$$
\begin{aligned}
& =g_{m_{\mathrm{eff}}} V_{\mathrm{bb}} \sin \omega_{\mathrm{bb}} t \cdot \mathrm{sq}\left[\sin \omega_{\mathrm{LO}} t\right] \\
& =g_{m_{\mathrm{eff}}}\left(\frac{4}{\pi}\right) V_{\mathrm{bb}} \sin \omega_{\mathrm{bb}} t\left[\sin \omega_{\mathrm{LO}} t+\frac{1}{3} \sin 3 \omega_{\mathrm{LO}} t+\cdots\right],
\end{aligned}
$$

where $s q\left[\sin \omega_{\mathrm{LO}} t\right]$ is the square wave function of LO signal and $g_{m_{\text {eff }}}$ is the effective transconductance of the mixer. The differential mixer output signal is eventually obtained as

$$
\begin{aligned}
V_{\text {out }}= & \frac{2}{\pi} R_{L} g_{m_{\mathrm{eff}}} V_{\mathrm{bb}} \\
& \times\left[\cos \left(\omega_{\mathrm{LO}}-\omega_{\mathrm{bb}}\right) t-\cos \left(\omega_{\mathrm{LO}}+\omega_{\mathrm{bb}}\right) t+\cdots\right]
\end{aligned}
$$
be

From (7), the conversion gain of the mixer is observed to

$$
\mathrm{CG}=\frac{V_{\text {out }}}{V_{\text {in }}}=\frac{2}{\pi} R_{L} g_{m_{\text {eff }}} .
$$

Looking at low voltage perspective, the derivation of overdrive voltage for conventional mixer as in Figure 1 is given as follows:

$$
V_{\mathrm{dd}}=V_{\mathrm{ds}_{1}}+V_{\mathrm{ds}_{3}}+V_{\mathrm{ds}_{7}}+V_{\mathrm{ds}_{9}}+V_{\mathrm{th}_{\succ 7}}
$$

where $V_{\mathrm{ds}}$ is the overdrive voltage and $V_{\text {th }}$ is the threshold voltage. Similarly, the supply voltage derived for the proposed mixer in Figure 2 can be derived as

$$
V_{\mathrm{dd}}=V_{\mathrm{ds}_{1}}+V_{\mathrm{ds}_{5}}+V_{\mathrm{ds}_{9}}+V_{\mathrm{th}_{\lrcorner_{1}}} .
$$

Equation (10) shows that the proposed mixer requires one less overdrive voltage of a transistor for the operation, which leads to reduced DC power consumption.

\section{Simulation Results}

The proposed architecture of the upconversion mixer is simulated and verified using Cadence Spectra RF platform. Components parameters are shown in Table 1. The design is implemented in a $0.13 \mu \mathrm{m}$ standard CMOS technology at supply headroom of $1.2 \mathrm{~V}$. The results verify that the upconversion mixer consumes a total current of $594 \mu \mathrm{A}$ which results in a dissipation of DC power of $0.71 \mathrm{~mW}$. Low DC power consumption is essentially vital in the application of wireless medical device such as capsule endoscope.

Figure $3(\mathrm{a})$ shows the input referred $1 \mathrm{~dB}$ compression point of $-5.43 \mathrm{dBm}$ and output referred $1 \mathrm{~dB}$ compression point of $-0.5 \mathrm{dBm}$. Two-tone analysis is conducted to verify the linearity characteristic as illustrated in Figure 3(b). The input third-order intercept point (IIP3) of $2.0 \mathrm{dBm}$ and output third-order intercept point (OIP3) of $7.1 \mathrm{dBm}$ were achieved with an equivalent gain of $5.4 \mathrm{~dB}$. This agrees with the general relationship of OIP3 = IIP3 + Gain, as also obtained in [1].

Table 2 enlists the performance comparison highlighting the proposed mixer respective to recent reported works. From Table 2, the proposed architecture highlights a lead in low power consumption and high linearity performance.
TABLE 1: Simulation parameters used for the proposed mixer of Figure 2.

\begin{tabular}{lc}
\hline Components & $W / L(\mu \mathrm{m})$ \\
\hline$M_{1}, M_{2}$ & $15 / 0.13$ \\
$M_{3}, M_{4}$ & $30 / 0.13$ \\
$M_{5}, M_{6}, M_{7}, M_{8}$ & $35 / 0.13$ \\
$M_{9}, M_{10}$ & $130 / 0.35$ \\
$M_{11}$ & $10 / 0.35$ \\
\hline$R_{L}$ & $1.7 \mathrm{k} \Omega$ \\
\hline
\end{tabular}

TABLE 2: Performance comparison.

\begin{tabular}{lccccc}
\hline & {$[8]^{*}$} & {$[9]^{* *}$} & {$[10]^{*}$} & {$[11]^{* *}$} & This work \\
\hline$V_{\text {DD }}(\mathrm{V})$ & 1.2 & 1.2 & 3.0 & 3.3 & 1.2 \\
Frequency $(\mathrm{GHz})$ & 4.0 & 23 & 2.45 & 0.9 & 0.434 \\
Gain $(\mathrm{dB})$ & 2.3 & 0.7 & 12.2 & -3.7 & 5.4 \\
OIP3 $(\mathrm{dBm})$ & 5 & $\approx 4.8$ & 9 & 13.3 & 7.1 \\
OP $_{1 \mathrm{~dB}}(\mathrm{dBm})$ & - & $\approx-6.1$ & 1.7 & 5.3 & -0.5 \\
Power $(\mathrm{mW})$ & 7.1 & 8.0 & 34.2 & 29.7 & 0.71 \\
LO power $(\mathrm{dBm})$ & 2.0 & 3.0 & 8.0 & 0 & -5.0 \\
FOM & - & -1.11 & -12.50 & -14.39 & 5.31 \\
\hline
\end{tabular}

${ }^{*}$ Simulation results. ${ }^{* *}$ Measurements results.

Lumping together the performance parameter the comparison is given by a figure of merit (FOM):

$$
\mathrm{FOM}=10 \log \left[\frac{10^{\mathrm{G} / 20} \cdot 10^{\mathrm{OP}_{1 \mathrm{~dB}} / 20} \cdot(\operatorname{Freq}(\mathrm{GHz}) / 1 \mathrm{GHz})}{\left(P_{\mathrm{DC}}(\mathrm{mW}) / 1 \mathrm{~mW}\right) \cdot\left(10^{P_{\mathrm{LO}} / 10} / 1 \mathrm{~mW}\right)}\right]
$$

where $G$ is the mixer gain, $\mathrm{OP}_{1 \mathrm{~dB}}$ is the output referred $1 \mathrm{~dB}$ compression point, Freq is the operating frequency, $P_{\mathrm{DC}}$ is the total power consumption with baseband and LO signal, and $P_{\mathrm{LO}}$ is local signal power in $\mathrm{dBm}$. The FOM originates from the fact that gain and $\mathrm{OP}_{1 \mathrm{~dB}}$ contribute to better performance of a mixer. At the same time, power consumption should be kept as low as possible in measuring a superior performance. Operating frequency is taken into account to obtain a fair comparison with other designs of different operating frequencies. Referring to the FOM comparison in Table 2, the proposed mixer shows a superior overall performance with a FOM of 5.31. The physical layout of the circuit is illustrated in Figure 4.

\section{Conclusion}

This paper presented a low power, high linearity upconversion mixer for the portable medical devices at $433 \mathrm{MHz}$. The low power architecture integrates regulated bias with inverting switching input. Implemented in $0.13 \mu \mathrm{m}$ standard CMOS technology the mixer consumes supply headroom of $1.2 \mathrm{~V}$ while dissipating $0.71 \mathrm{~mW}$ of $\mathrm{DC}$ power. 


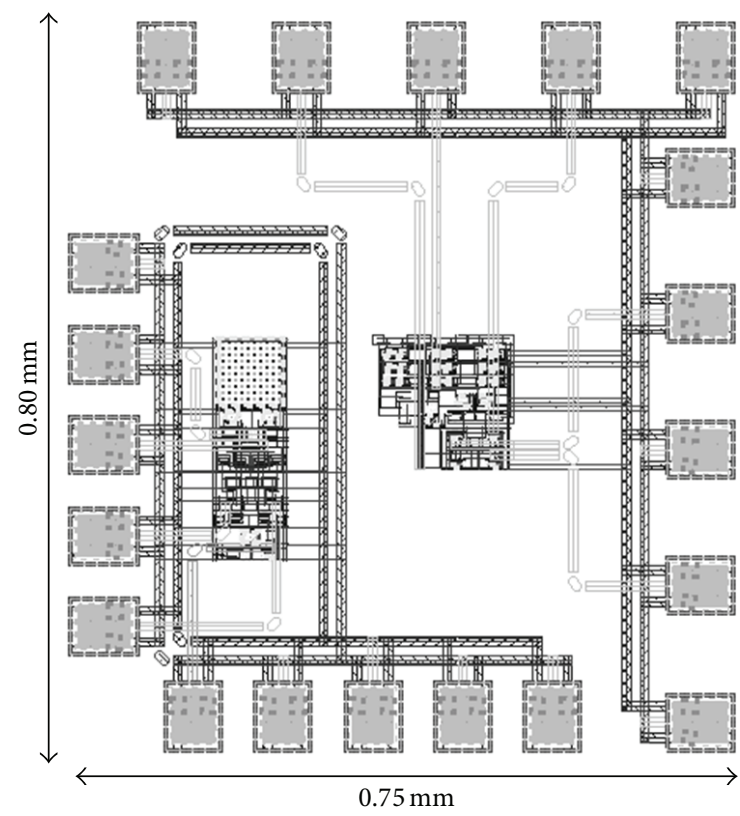

Figure 4: Layout of the proposed mixer.

\section{Conflict of Interests}

The authors declare that there is no conflict of interests regarding the publication of this paper.

\section{Acknowledgment}

The authors would like to thank the Ministry of Science, Technology and Innovation (MOSTI), Malaysia, for financially supporting this research under eScience Fund.

\section{References}

[1] V. Vidojkovic, J. van der Tang, A. Leeuwenburgh, and A. H. M. van Roermund, "A low-voltage folded-switching mixer in $0.18 \mu \mathrm{m}$ CMOS," IEEE Journal of Solid-State Circuits, vol. 40, no. 6, pp. 1259-1263, 2005.

[2] X. Wang and P. Andreani, "Comparison of the image rejection between the passive and the Gilbert mixer," in Proceedings of the IEEE International Symposium on Cirquits and Systems (ISCAS '04), vol. 1, pp. I-968-I-971, May 2004.

[3] F. Behbahani, Y. Kishigami, J. Leete, and A. A. Abidi, "CMOS mixers and polyphase filters for large image rejection," IEEE Journal of Solid-State Circuits, vol. 36, no. 6, pp. 873-887, 2001.

[4] E. A. M. Klumperink, S. M. Louwsma, G. J. M. Wienk, and B. Nauta, "A CMOS switched transconductor mixer," IEEE Journal of Solid-State Circuits, vol. 39, no. 8, pp. 1231-1240, 2004.

[5] G. Grau, U. Langmann, W. Winkler, D. Knoll, J. Osten, and K. Pressel, "A current-folded up-conversion mixer and VCO with center-tapped inductor in a SiGe-HBT technology for 5-GHz wireless LAN applications," IEEE Journal of Solid-State Circuits, vol. 35, no. 9, pp. 1345-1352, 2000.

[6] F. P. Cortes and S. Bampi, "A 1.4GHz upconversion mixer design using the gm/ID method suitable for a multi-band analog interface," in Proceedings of the 14th IEEE International
Conference on Electronics, Circuits and Systems (ICECS '07), pp. 1400-1403, Marrakesh, Morocco, December 2007.

[7] G. H. Tan, R. M. Sidek, H. Ramiah, and W. K. Chong, "Design of ultra-low voltage $0.5 \mathrm{~V}$ CMOS current bleeding mixer," IEICE Electronics Express, vol. 9, no. 11, pp. 990-997, 2012.

[8] S. A. Z. Murad, R. K. Pokharel, H. Kanaya, and K. Yoshida, "A $3.0-5.0 \mathrm{GHz}$ high linearity and low power CMOS upconversion mixer for UWB applications," in Proceedings of the IEEE International Conference of Electron Devices and SolidState Circuits (EDSSC '10), pp. 1-4, Hong Kong, December 2010.

[9] A. Verma, K. O. Kenneth, and J. Lin, "A low-power upconversion CMOS mixer for 22-29-GHz ultra-wideband applications," IEEE Transactions on Microwave Theory and Techniques, vol. 54, no. 8, pp. 3295-3300, 2006.

[10] J. Y. Choi and S. G. Lee, "A $2.45 \mathrm{GHz}$ CMOS up-conversion mixer design utilizing the current-reuse bleeding technique," International Journal of Electronics, vol. 91, no. 9, pp. 537-550, 2004.

[11] K. Choi, S. Yoo, M. Kim et al., "CMOS DSB transmitter with low TX noise for UHF RFID reader system-on-chip," IEEE Transactions on Microwave Theory and Techniques, vol. 58, no. 12, pp. 3467-3474, 2010.

[12] K. Kim, S. Yun, S. Lee, S. Nam, Y. J. Yoon, and C. Cheon, "A design of a high-speed and high-efficiency capsule endoscopy system," IEEE Transactions on Biomedical Engineering, vol. 59, no. 4, pp. 1005-1011, 2012. 

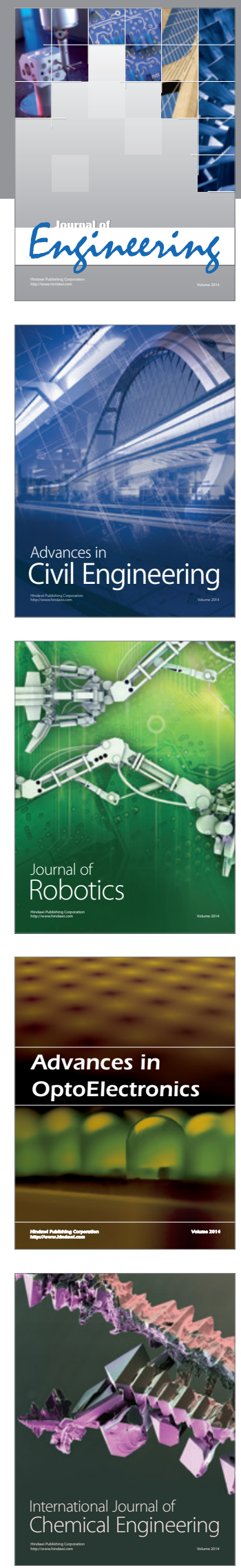

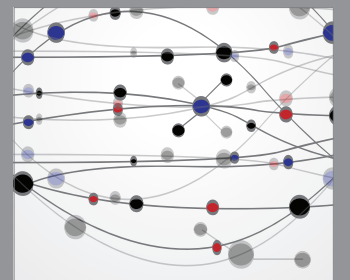

The Scientific World Journal
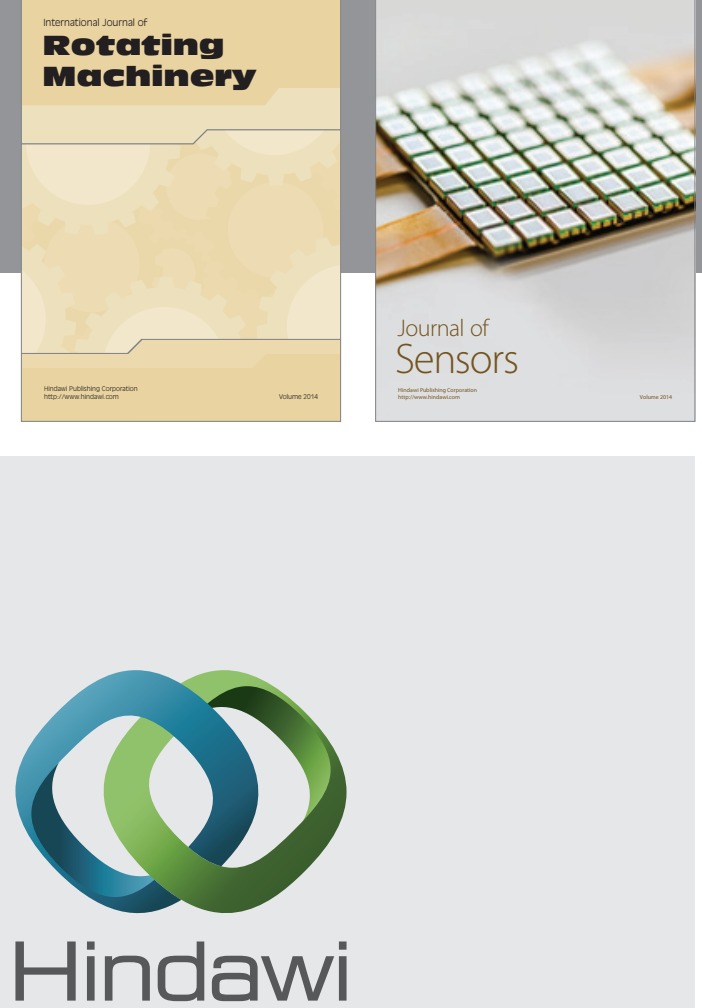

Submit your manuscripts at http://www.hindawi.com
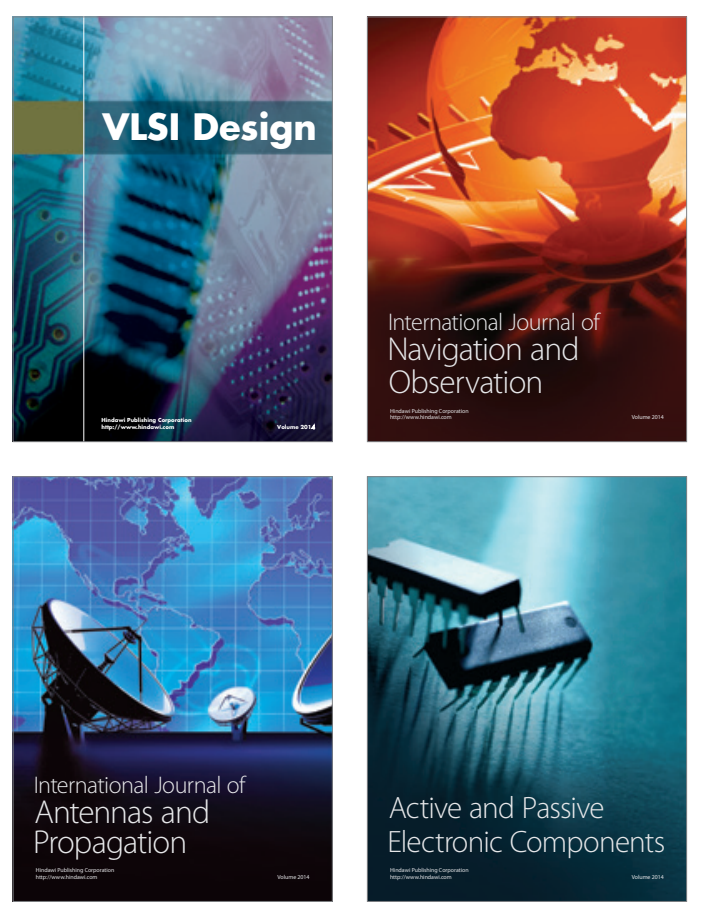
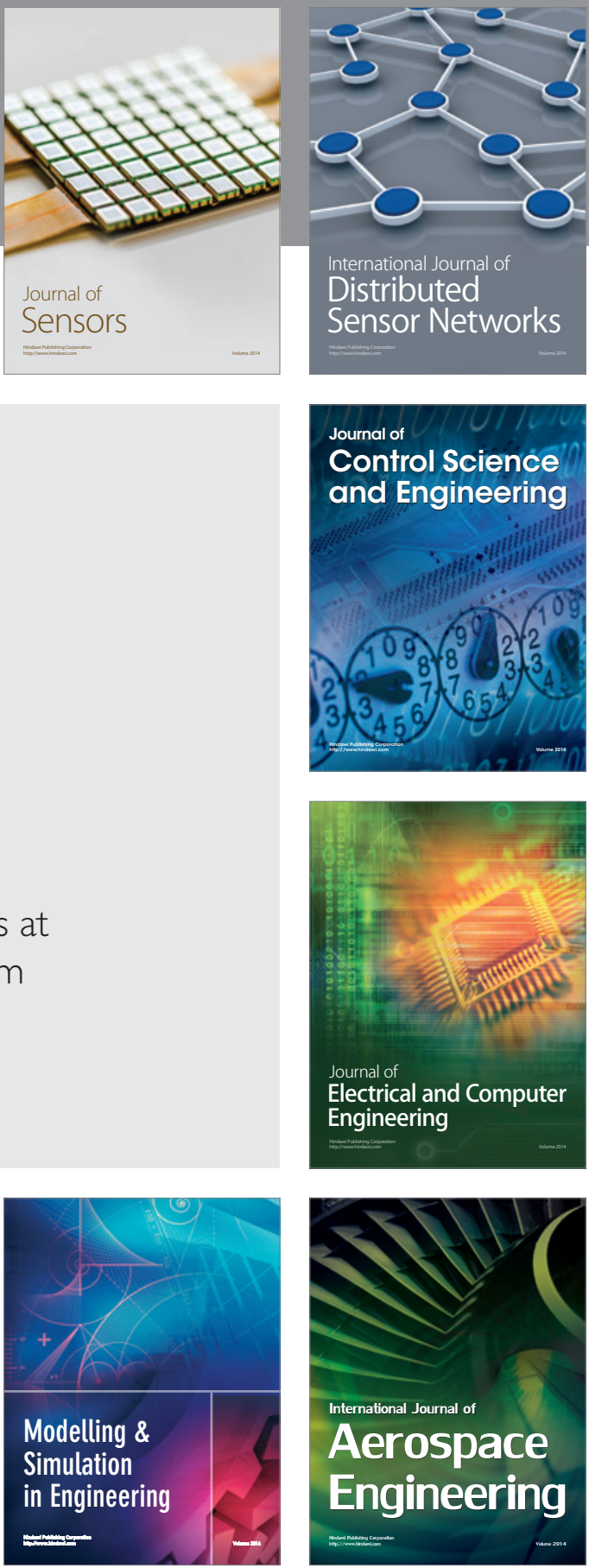

Journal of

Control Science

and Engineering
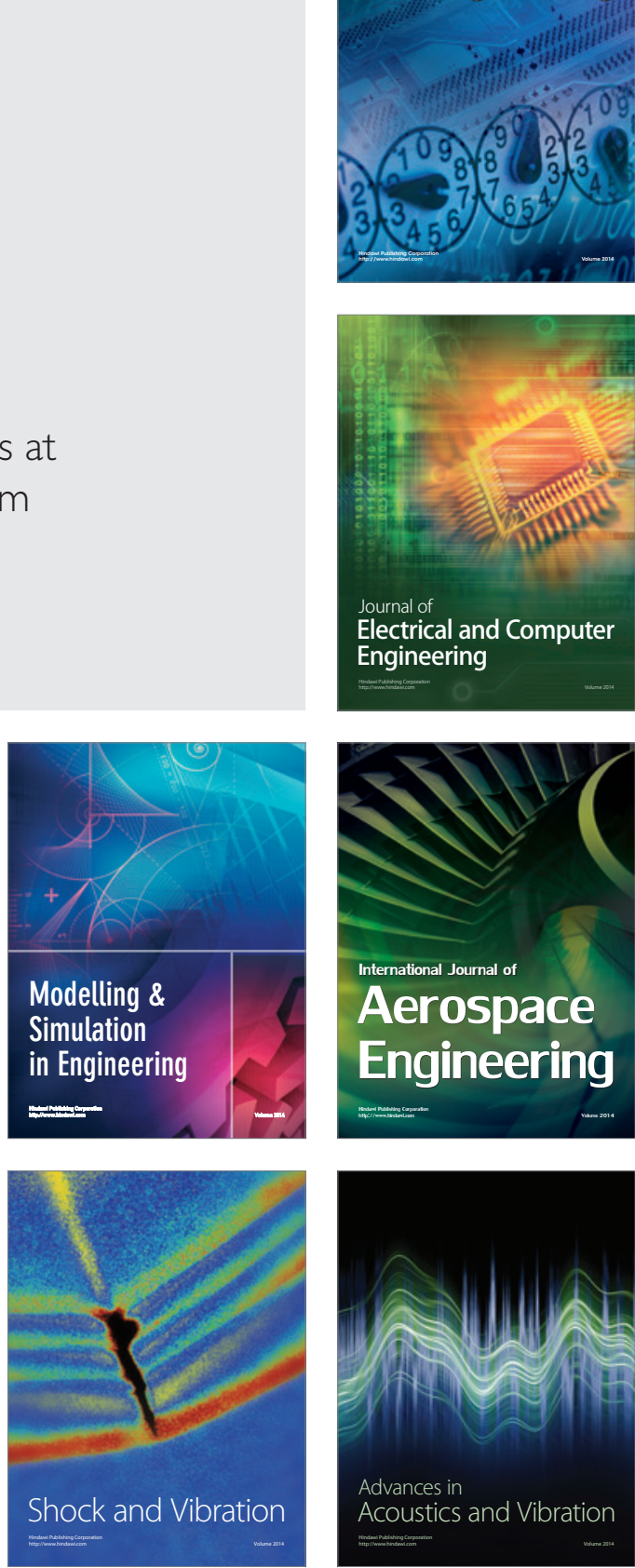